\title{
Case Series \\ ABDOMINAL TUBERCULOSIS IN NEPAL MEDICAL COLLEGE TEACHING HOSPITAL, KATHMANDU
}

\author{
Shrestha S. ${ }^{1}$, Pradhan G. B. ${ }^{2}$, Bhoomi K. ${ }^{3}$, Shrestha B. L. ${ }^{4}$, Bhattachan C. L. ${ }^{4}$ \\ ${ }^{1}$ Assistant Prof., ${ }^{2}$ Lecturer, ${ }^{3}$ Assistant Prof, Department of Medicine, ${ }^{4}$ Pro., Head of Department, Department of \\ Surgery, Nepal Medical College Teaching Hospital, Attarkhel, Jorpati, Kathmandu
}

\begin{abstract}
Abdominal Tuberculosis is a common extra pulmonary manifestation of tuberculosis. The wide spectrum of presentation makes abdominal tuberculosis difficult to diagnose and treat. Retrospective review of cases with abdominal tuberculosis presenting to the Surgery Department of Nepal Medical College Teaching Hospital from January 2002- June 2007 was done to describe our experience of abdominal tuberculosis over a 5 year period.
\end{abstract}

We found total 32 patients with abdominal tuberculosis, among which 13 had concurrent pulmonary tuberculosis. The most common clinical presentation, i. e. a triad of abdominal pain, fever and weight loss was present in 13 who had symptoms of pulmonary tuberculosis presented to physicians and the remaining 19 presented acutely to surgeons with symptoms of pain and obstruction. Chest $X$ ray, abdominal ultrasound and barium meal follow through done to find associated abnormalities. Diagnostic Laparoscopy was performed in 10 and 3 patients with peritonitis underwent emergency laparotomy which revealed multiple ileal perforations in 2 cases and one had multiple strictures with small bowel perforation. Diagnosis of tuberculosis, attended at surgeons was confirmed by demonstrating caseating granulomas in histology and Acid Fast Bacilli Positive, culture for M. tuberculosis from peritoneal fluid. All patients were started anti tuberculosis treatment.

Abdominal tuberculosis is a relatively common finding and should always be considered in the differential diagnosis of abdominal pain, fever and weight loss.

Key Words: Tuberculosis; abdominal tuberculosis; Acid Fast Bacilli; ileocaecal disease

\section{Introduction}

Between 1980-2005, 90 million TB patients were registered in national surveillance systems and reported to WHO globally. The global TB incidence rate peaked sometime between 2000-2005, although the total number

\section{Correspondence to:}

Dr. Sunil Shrestha, Assistant Professor

Department of Surgery, Nepal Medical College

Teaching Hospital

Email: phoolbari@yahoo.com of new cases is still raising each year. ${ }^{1}$ One-half of the world population is infected with $M$. tuberculosis, and it is the leading cause of infectious death, with approximately 1.6 million deaths annually. ${ }^{2}$

Abdominal tuberculosis is one of the most prevalent forms of extra-pulmonary tuberculosis disease. The Gastro Intestine (GI) tract, peritoneum, lymphatic system, and solid viscera are subject to differing degrees of tuberculosis involvement, which can occur 
alone or in combination. Tuberculosis has a wide spectrum of illness and can affect any system in the body. Tuberculosis of $\mathrm{Gl}$ tract accounts for $50 \%$ of all gastro intestinal cases. The commonest site is distal ileum and caecum. $^{3}$ Abdominal tuberculosis is an important but probably underestimated clinical problem.

The diagnosis of abdominal tuberculosis is often delayed, increasing the morbidity associated with this treatable condition. In this case series, the clinical presentations and outcomes of 32 patients with abdominal tuberculosis were reviewed retrospectively. Our aim was to elucidate the presenting signs and symptoms of abdominal tuberculosis to make the diagnosis and treatment in early.

\section{Methodology}

This is hospital record based case series study, which is carried out retrospectively, for 5 year period from January 2002 to June 2007 in Nepal Medical College Teaching Hospital, Jorpati which is one of the tertiary care hospitals of Katmandu, Nepal. During study period 32 cases of abdominal tuberculosis were notified.

Diagnoses of abdominal tuberculosis were based on one of these criteria: 1) characteristic caseating granulomas in histologic specimens; 2) GI symptoms combined with abdominal ultrasound showing abnormalities of the peritoneum and bowel that improved with antituberculous treatment; and 3) Patients with symptoms of clinical triad of abdominal pain, fever, and weight loss associated with past history of pulmonary tuberculosis.

\section{Results}

Thirty-two cases of abdominal tuberculosis were identified in the period January 2002 to June 2007. Median age of the patients was 38 years (range, 14-81 years), 22 (68.7\%) were females and 10 (31.3\%) were males.

Thirteen patients (40.6\%) who had symptoms of pulmonary tuberculosis with associated abdominal tuberculosis were presented to physicians and the remaining nineteen patients presented acutely to surgeons with symptoms of pain and obstruction. The most common clinical presentation was a triad of abdominal pain (85.0\%), fever (78.6\%) and weight loss $(66.5 \%)$. The abdominal pain was of varying quality, most frequently cramping in nature. In most cases fever was low grade and was associated with general weakness and malaise. Nausea, vomiting and diarrhea were also present in more than half of the patients. (Table1) Duration of symptoms ranged from 15 days to almost 18 months (mean 3.5 months). Of the 32 patients tested by Mantoux test, 17 (53.1\%) were positive.

\section{Table 1 Clinical Presentation in 32 Cases of} Abdominal Tuberculosis

\begin{tabular}{|l|l|}
\hline Clinical feature & Numbers (\%) \\
\hline Abdominal pain & $27(85.0 \%)$ \\
\hline Fever & $24(78.6 \%)$ \\
\hline Weight Loss & $21(66.5 \%)$ \\
\hline Cough & $13(40.6 \%)$ \\
\hline Diarrhea & $15(46.0 \%)$ \\
\hline Ascites & $11(34.3 \%)$ \\
\hline
\end{tabular}

Laboratory investigations revealed low hemoglobin level in 60\% cases and more than 90\% had elevated C-reactive protein and Erythrocyte Sedimentation Rate.

Chest X-ray was abnormal with calcified granulomas in 13 (40.6\%) cases, who had completed the course of anti tuberculosis treatment due to previous history of pulmonary tuberculosis and in $3(9.3 \%)$ cases there were free gas under the diaphragm suggestive of hollow viscus perforative peritonitis that was confirmed clinically. Abdominal ultrasound was done in all patients and it showed free fluid in 
the peritoneal cavity in $11(34.3 \%)$ cases, thickened small bowel wall in 8(25.0\%) cases and mesenteric lymphadenopathy in 6(18.7\%) cases. Twenty two patients underwent barium meal follow through of whom 15(71.4\%) had small bowel strictures and the rest were normal.(Table 2)

Table 2 Radiological Findings in 32 Cases of Abdominal Tuberculosis

\begin{tabular}{lll}
\hline $\begin{array}{l}\text { Imaging } \\
\text { Modality }\end{array}$ & $\begin{array}{l}\text { Number of } \\
\text { cases (\%) }\end{array}$ & \multicolumn{1}{c}{ Findings } \\
\hline $\begin{array}{l}\text { Chest } \\
\text { X-ray }\end{array}$ & $13(40.6 \%)$ & Calcified granulomas \\
$\begin{array}{l}\text { Ultrasound } \\
\text { abdomen }\end{array}$ & $11(34.3 \%)$ & $\begin{array}{l}\text { Free gas under diaphragm } \\
\text { peritoneal cavity }\end{array}$ \\
$\begin{array}{l}\text { Barium meal } \\
\text { follow }\end{array}$ & $15(71.4 \%)$ & $\begin{array}{l}\text { Small bowel Stricture } \\
\text { through }\end{array}$ \\
\hline
\end{tabular}

Diagnostic Laparoscopy was performed in Ten $(31.2 \%)$ cases out of which Six $(60.0 \%)$ had mesenteric lymphadenitis and in 4 patients there was serosal infiltration of tubercles in the entire small bowel which confirmed granulomatous lesions in histology. In all eleven patients with ascites, fluid analysis revealed elevated white blood cell counts. The ascitic fluid protein was $>5 \mathrm{gm} / \mathrm{dl}$ in all patients. AcidFast Bacilli were not seen in any of the ascitic fluid smears but the culture was positive in 6 (54.5\%) cases. Three patients with peritonitis underwent emergency laparotomy which revealed multiple ileal perforations in 2 cases and one patient had multiple strictures with small bowel perforation. In one patient long segment ileal resection with proximal ileostomy was performed. The other patient had mid ileal resection with end to end anastomosis and the last one undergone segmental ileal resection, stricturoplasty with enterocolic bypass. The later two patients died post operatively due to severe acute respiratory distress syndrome.

All 13 patients who were diagnosed by tissue biopsy from peritoneum, mesenteric nodes, omental tissue and serosal tissue, the another 13 patients diagnosed presumptively by the presence of previous history of pulmonary TB in combination with signs, symptoms, and radiographic evidence for abdominal tuberculosis and the six patients diagnosed with AFB culture from ascetic fluid received anti tuberculous treatment for 6-12 months. All the patients were treated with initial 2 month phase with rifampicin, isoniazid, pyrazinamide and ethambutol and then maintenance phase with rifampicin and isoniazid.

Overall, four (13.3\%) patients relapsed with recurrent episode of sub acute bowel obstruction 2 years after successful completion of therapy for abdominal tuberculosis. Twenty eight patients were followed up for an average period of 12 months. (Range, 3-24 months)

\section{Discussion}

Up to $5 \%$ of patients with $M$. tuberculosis have $\mathrm{GI}$ involvement, and the $\mathrm{GI}$ tract is reported to be the sixth most common extra pulmonary site. ${ }^{4}$ Of those with $\mathrm{GI}$ Tract Tuberculosis, up to $60 \%$ may have active pulmonary TB. ${ }^{5}$

In keeping with the published data ${ }^{6,7,8}$ fever was the commonest presenting feature in our study with weight loss, abdominal pain and malaise present in greater than $80 \%$ cases. Past history of treatment for pulmonary TB was present in $40 \%$ of our cases and it is likely that decreased immunity caused reactivation of TB foci.

Our results suggest that abdominal tuberculosis is often difficult to diagnose. The signs and symptoms are nonspecific, and in our sample, clinicians often failed to consider TB in the initial differential diagnosis.

Laparoscopy with biopsy remains the gold standard in diagnosis of peritoneal TB, with a reported sensitivity of $100 \%$ and a low 
complication rate. ${ }^{8}$ Other studies have suggested that smear of the peritoneal fluid for AFB is rarely positive. ${ }^{4}$ Culture positivity varies from $20-83 \%{ }^{4}$ In our study 11 patients with ascites, all had negative AFB smears and $6(54.5 \%)$ had positive cultures for $M$. tuberculosis.

Similar laboratory findings in relation to level of haemoglobin, C-reactive protein and ESR were found in other studies like Marshall $\mathrm{JB}^{4}$ and Probert CJ. ${ }^{5}$

Four of the seven patients with enteric TB had involvement of the terminal ileum and/or cecum. This is consistent with other studies that show enteric TB most frequently involving the ileocecal area. This tropism is thought to be due to the relative physiologic stasis in this area and to the increased density of lymphatic tissue, for which the bacilli have an affinity. 9,10,11

TB can affect any region of the gut from esophagus to rectum but the ileo-caecal region is the most commonly involved.12 This was true in our series as well with ileocaecal region in $57.2 \%$ cases followed by peritoneal involvement in $41.4 \%$ cases being the commonest sites to be affected. Laparoscopic biopsies, being larger in size and visually directed, can provide histological evidence in $70-85 \%$ cases $^{13}$ In this series it was performed in $10(31.2 \%)$ cases and not only provided the diagnosis in elusive cases but also helped to ascertain the extent of intra-abdominal spread of TB.

In summary, abdominal tuberculosis is a disease that is frequently overlooked, with consequent delay in treatment. As a treatable disorder, abdominal TB should be considered early in the differential diagnosis of abdominal symptoms. Failure to recognize this disease early may lead to increased morbidity and mortality.

\section{References}

1. Global tuberculosis Control, WHO Report 2007, WHO

2. Chan ED, Iseman MD. Current medical treatment for tuberculosis. Br Med J 2002; 325:1282-1286

3. National Tuberculosis Programme Of Nepal, a Clinical Manual, 2005

4. Marshall JB. Tuberculosis of gastrointestinal tract and peritoneum. Am J Gastroenterol 1993;88:989-99

5. Probert CJ, Jayanthi V, Wicks AC et el. Epidemiological study of abdominal tuberculosis among Indian migrants and the indigenous population of Leicester. Gut 1992; 33:1085-1088.

6. Singhal, Amit, Gulati et el . Abdominal tuberculosis in Bradford, UK: 1992-2002, Europian journal of gastroenterology and hepatology Volume 17(9), September 2005, pp 967-971

7. Sherman S, Rohwedder JJ, Ravikrishnan KP, Weg JG. Tuberculous enteritis and peritonitis: report of 36 general hospital. Arch Intern Med 1980;140:506-8.

8. Bernhard JS, Bhatia G, Knauer CM. Gastrointestinal tuberculosis: an eighteen patient experience and review. J Clin Gastroenterol 2000; 30:397-402.

9. Jabukowski, A, Elwool RK, Enaison DA. Clinical features of abdominal tuberculosis. J Infect Dis 1988;158:687-92.

10. Sherman S, Rohwedder JJ, Ravikrishnan KP, Weg JG. Tuberculous enteritis and peritonitis: report of 36 general hospital. Arch Intern Med 1980;140:506-8.

11. Bernhard JS, Bhatia G, Knauer CM. Gastrointestinal tuberculosis: an eighteen patient experience and review. J Clin Gastroenterol 2000; 30:397-402

12. Marshall JB. Tuberculosis of the gastrointestinal tract and peritoneum. Am J Gastroenterol 1993; 88:989-999

13. Lisehora GB, Peters CC, Lee YTM, Barcia PJ. Tuberculous peritonitis-do not miss it. Dis Colon Rectum 1996;39:394-9. 